\title{
Work-in-Progress: Computation Offloading of Acoustic Model for Client-Edge-Based Speech-Recognition
}

\author{
Young-Min Lee*ף \\ *Department of Semiconductor and Display \\ Engineering, Sungkyunkwan University, Suwon, Korea \\ qSamsung Electronics Co., Ltd., Hwaseong, Korea \\ ymgoon.lee@skku.edu
}

\begin{abstract}
Speech recognition technology combined with artificial intelligence represents a quantum leap more accurate than past pattern recognition methods. And server-based system support for scalability, virtualization and huge amounts of unlimited storage resources that greatly contributed to the improvement of the accuracy of its prediction. However, the implementation of server-oriented reforms led to enormous latency and connectivity problems. Therefore, we propose a novel client-edge speech recognition system to enhance latency by using what we call semi-offloading technology. This proposal is promising big performance gains by offloading computing power-dependent tasks to edge nodes and processing throughput-dependent tasks by a client. The merit of semi-offloading as well as a division of workload allows for parallelism and re-ordering among the process. The experimental results show that, $23 \% \sim 62 \%$ improvement in response time.
\end{abstract}

\section{CCS CONCEPTS}

- Networks $\rightarrow$ Location based services; • Computing methodologies $\rightarrow$ Speech recognition.

\section{KEYWORDS}

Speech-Recognition, Semi-Offloading, Edge Computing

\section{INTRODUCTION}

The study of speech recognition is a well-respected field. Because it is a revolutionary technology that could replace the standardized keyboard or touch system for entering commands. And it has grown rapidly on the basis of a cloudcomputing and deep-learning technology that providing great computing power and huge storage capacity. Nevertheless, this server-based speech recognition system has latency and

Permission to make digital or hard copies of all or part of this work for personal or classroom use is granted without fee provided that copies are not made or distributed for profit or commercial advantage and that copies bear this notice and the full citation on the first page. Copyrights for components of this work owned by others than ACM must be honored. Abstracting with credit is permitted. To copy otherwise, or republish, to post on servers or to redistribute to lists, requires prior specific permission and/or a fee. Request permissions from permissions@acm.org.

CASES'19 Companion, October 13-18, 2019, New York, NY, USA

(C) 2019 Association for Computing Machinery.

ACM ISBN 978-1-4503-6925-1/19/10. .\$15.00

https://doi.org/10.1145/3349569.3351534

\author{
Joon-Sung Yang* \\ *Department of Semiconductor and Display \\ Engineering, Sungkyunkwan University, Suwon, Korea \\ js.yang@skku.edu
}

connectivity problems that must be solved. Digalakis [1] showed that problem and sorted system into three forms as follows server-only processing, client-only processing, and client-server processing. This paper presents a novel clientedge processing system that combined an emerging edgecomputing to a deep learning process of automatic speech recognition. Unlike preceding studies, this paper refines the procedures consists of four major activities as follows analogto-digital convert, feature extraction, acoustic model processing, and language model processing. These procedures are redistributed to a client and edge node base on computing power or processing capacity of data. This study will focus primarily on comparing server-only processing and client-edge processing.

Table 1: Speech Recognition System Configuration

\begin{tabular}{|c|c|c|c|}
\hline \multirow{3}{*}{$\begin{array}{l}\text { Distributed } \\
\text { Processing Models }\end{array}$} & \multicolumn{3}{|c|}{ Processing tasks \& Locations } \\
\hline & Client & Edge & Server \\
\hline & Node & Node & Node \\
\hline server-only & $\tau$ & - & $\mu \alpha \ell$ \\
\hline client-only & $\tau \mu \alpha \ell$ & - & - \\
\hline client-server & $\tau \mu$ & - & $\alpha \ell$ \\
\hline client-edge & $\tau \mu \ell$ & $\alpha$ & - \\
\hline
\end{tabular}

\section{ANALOG TO DIGITAL CONVERT}

$\mathrm{ADC}$ is a device observing variable analog audio signals at its input and producing a digital wave file at its outputs. Background noise is reduced and speech signals are quantized into binary words at the sampling time.

\section{FEATURE EXTRACTION}

$\mathrm{FE}$ is to derive a vector. It has a very similar value for the same phoneme. Therefore, the vector can be used to represent a phoneme. Among lots of $\mathrm{FE}$ technique, we use MFCC technique in this paper.

\section{ACOUSTIC \& LANGUAGE MODEL}

$\mathrm{AM}$ takes as an input feature vector, and predict outputs probabilities. Each probability indicates the likelihood that any given frame belongs to phoneme. LM measures the probability of word and phrase in the target language. The weight 
data required for the prediction. And the size of AM weight data is specified from $50 \mathrm{OMB}$ to $200 \mathrm{MB}$ and the size of $\mathrm{LM}$ weight data is specified from $10 \mathrm{~GB}$ to $100 \mathrm{~GB}$ regarding one language does not compress.

\section{PROPOSED ARCHITECTURE}

The feature of this proposal is while each ADC, FE, and LM is processed by a client, AM is delivered to the edge node. We call this semi-offloading. The reason for semi-offloading is AM has eight-time computing workloads more than LM and typical server speed is superior to client speed and a client has full LM capability. The CPU speed of Intel Xeon-phi is @ $96 \mathrm{GHz}$ (1.5 GHz*64Threads, 64 Cores) in a different class from the latest Samsung galaxy s1o whose CPU speed is @ $17.6 \mathrm{GHz}$ $\left[(2.7 \mathrm{GHz}+2.3 \mathrm{GHz}) *{ }_{2}\right.$ Threads $+1.9 \mathrm{GHz}^{*} 4$ Threads, 6Cores $]$. Beyond that, semi-offloading takes advantage of response time, latency and battery life. In addition, it can reduce overhead such as protocol handling during up/downstream. Moreover, it also allows for parallelism and re-ordering between LM loading and request. i.e., we can load AM weight data not waiting until response/request is received.

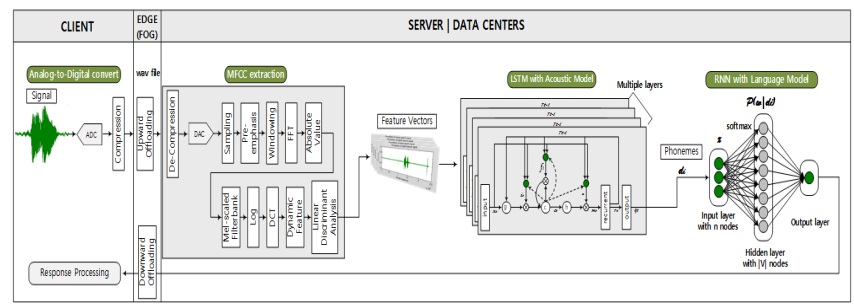

(a) Conventional Server-Only Speech Recognition System

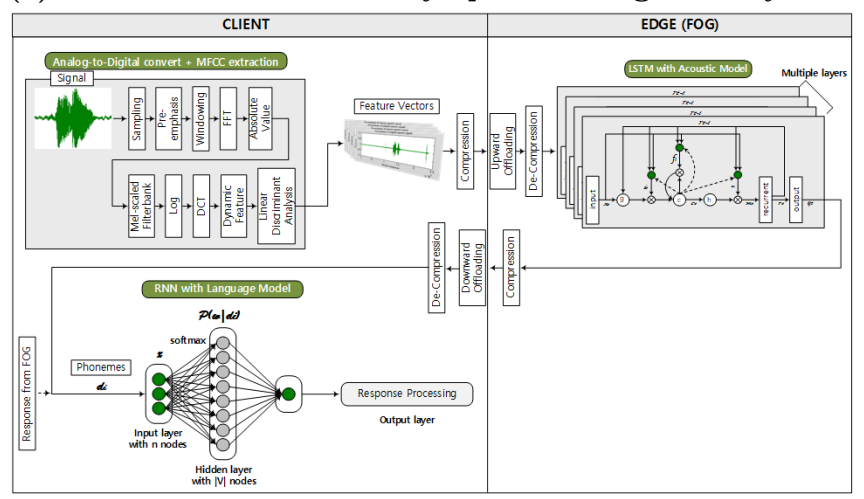

(b) Proposed Client-Edge Speech Recognition System

Figure 1: System Diagram of Speech Recognition System 6 EVALUATION

For experiments, the audio encoder and decoder of 16 -bit resolution and $16 \mathrm{kHz}$ sampling clock is used. MFCC is implemented to extract the coefficients. AM is composed of 8 LSTM layers,1 fully connected layer and CTC set with ${ }_{153} \mathrm{MB}$ trained weight data to identify a single alpha character. LM uses $10 \mathrm{~GB}$ trained weight data from the training set of 500 hours of speech provided by Open Speech and Language Resources. The CPU speed of edge modeling is
@ $43.2 \mathrm{GHz}$ using Intel Xeon-e5-i650 V4 (3.6 GHz*12Threads, 6Cores). We use Google cloud speech-to-text API to assess a conventional server-based speech recognition system. The test configuration unit is a sentence that is composed of 30 words. And the test is repeated five times from one sentence to twenty-five sentences. The experimental results showed that response time is improved by $23 \% \sim 62 \%$ compared to a server-only model by semi-offloading at the simulation condition of increasing sentence number. And the min-max variation of response time was similar to a client-only model and was stable.

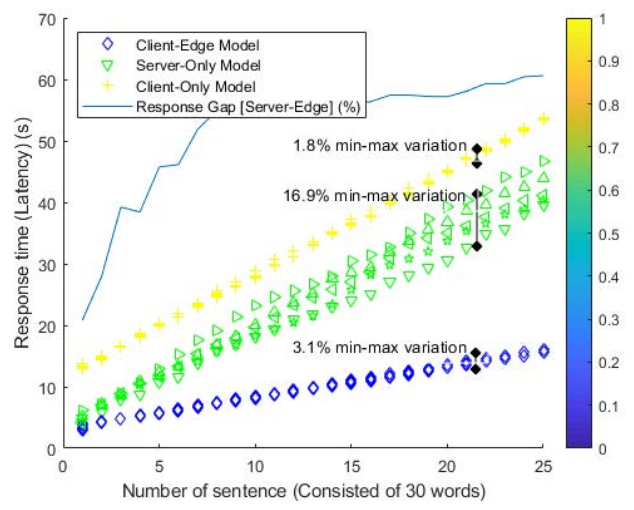

Figure 2: Response Times of Speech Recognition System

\section{CONCLUSION}

We demonstrated the problem as server-based speech recognition and also designed a client-edge-based system. Through evaluation, we discovered low variability of response time more than a server-based system on amounts of utterance. We found the optimization point of operation pipeline and the potential of greatly reducing latency.

\section{ACKNOWLEDGMENTS}

This work was supported in part by the Institute of Information \& communications Technology Planning \& Evaluation (IITP) grant funded by the Korea government (MSIT) (No.2019-0-00421, AI Graduate School Support Program), and in part by the Basic Science Research Program through the National Research Foundation of Korea by the Ministry of Education under Grant NRF-2018R1D1A1Bo7049842.

\section{REFERENCES}

[1] Digalakis, V. V., Neumeyer, L. G., And Perakakis, M. Quantization of cepstral parameters for speech recognition over the world wide web. IEEE Journal on selected areas in communications 17, 1 (1999), 82-90.

[2] Hain, T., Christian, J., Saz, O., Deena, S., Hasan, M., Ng, R. W., Milner, R., Doulaty, M., And LiU, Y. webasr 2-improved cloud based speech technology. In INTERSPEECH (2016), pp. 16131617 .

[3] McGraw, I., Prabhavalkar, R., Alvarez, R., Arenas, M. G., Rao, K., Rybach, D., Alsharif, O., Sak, H., Gruenstein, A., Beaufays, F., ET AL. Personalized speech recognition on mobile devices. In 2016 IEEE International Conference on Acoustics, Speech and Signal Processing (ICASSP) (2016), IEEE, pp. 5955-5959. 